\title{
Does gender influence the impact of impaired renal function on prognosis after ST-segment elevated myocardial infarction?
}

\author{
Joon Seok Choi ${ }^{1}$, Min Jee Kim ${ }^{1}$, Yong Un Kang ${ }^{1}$, Chang Seong Kim ${ }^{1}$, Eun Hui Bae ${ }^{1}$, \\ Seong Kwon $\mathrm{Ma}^{1}$, Young-Keun $\mathrm{Ahn}^{1,2}$, Myung Ho Jeong ${ }^{1,2}$, Young Jo Kim³ \\ Myeong Chan $\mathrm{Cho}^{4}$, Chong Jin $\mathrm{Kim}^{5}$, Soo Wan Kim ${ }^{1}$; \\ other Korea Acute Myocardial Infarction Registry Investigators* \\ ${ }^{1}$ Department of Internal Medicine, Chonnam National University Medical School, Gwangju, Korea \\ ${ }^{2}$ Cardiovascular Research Institute of Chonnam National University, Gwangju, Korea \\ ${ }^{3}$ Department of Internal Medicine, Yeungnam University, Daegu, Korea \\ ${ }^{4}$ Department of Internal Medicine, Chungbuk National University, Cheongju, Korea \\ ${ }^{5}$ Department of Internal Medicine, Kyunghee University, Seoul, Korea
}

\begin{abstract}
Background: A limited number of studies have investigated the impact of gender on renal function and clinical outcomes after ST-segment elevated myocardial infarction (STEMI), and these studies have provided discrepant results.

Methods and Results: This study was based on a retrospective cohort, the Korean Acute Myocardial Infarction Registry (KAMIR). Patients $(n=7,679)$ with a discharge diagnosis of STEMI were analyzed to investigate association of gender with renal function and clinical outcomes. Compared to men, women were older and exhibited more comorbidity, including impaired renal function. Women showed higher mortality compared to men (1-month mortality, $5.6 \%$ in men vs. $12.6 \%$ in women, $p<0.001$; 1-year mortality, $6.8 \%$ in men vs. $14.4 \%$ in women, $p<0.001)$. The risk of death proportionally increased as estimated glomerular filtration rate (eGFR) decreased in both genders. After adjusting for potential confounders, hazard ratios for women did not significantly differ from those for men at each eGFR level. The interaction test showed no significant interaction between gender and eGFR in 1-month mortality and 1-year mortality.

Conclusions: Impaired renal function was an independent prognostic factor after STEMI in both genders, and the impact of impaired renal function on prognosis after STEMI did not significantly differ between genders. (Cardiol J 2013; 20, 5: 526-532)

Key words: gender, impaired renal function, ST-segment elevated myocardial infarction
\end{abstract}

\footnotetext{
Address for correspondence: Soo Wan Kim, MD, PhD, Department of Internal Medicine, Chonnam National University Medical School, 42 Jebongro, Gwangju 501-757, Korea, tel: +82-62-220-6271, fax: +82-62-225-8578, e-mail: skimw@chonnam.ac.kr

*Korea Acute Myocardial Infarction Registry Investigators are listed in Appendix 1.

Received: 07.11.2012

Accepted: 15.02.2013
} 


\section{Introduction}

Impaired renal function is a well-known independent risk factor for mortality and morbidity in patients with myocardial infarction (MI) [1, 2]. Several studies reported that impaired renal function is more prevalent in women, while female MI patients are generally older and have more comorbidity than male patients $[2,3]$. The impact of gender difference on prognosis after ST-segment elevated MI (STEMI) has been a popular and debated topic in clinical practice [4-10]. Although many studies on the issue have consistently showed higher mortality in women compared with men after STEMI [4-10], a limited number of studies have investigated gender influence on the prognostic impact of impaired renal function in patients with STEMI. Moreover, published studies yielded discrepant results regarding the impact of gender on the relationship between renal function and clinical outcomes in STEMI patients [11, 12]. Therefore, we undertook the present study to investigate how gender influences the prognostic impact of impaired renal function in patients with STEMI, in a cohort registered in the Korea Acute Myocardial Infarction Registry (KAMIR).

\section{Methods}

\section{Korea Acute Myocardial Infarction Registry}

The study population was derived from the KAMIR. The KAMIR is a Korean prospective, open, observational, multi-center online registry investigating the risk factors of mortality in MI patients since November 2005. Details of the KAMIR have been published elsewhere $[2,13]$. We assessed a cohort of 8,063 consecutive patients who were admitted to the hospital between November 1,2005 , and July 31,2008 , and had a discharge diagnosis of STEMI, confirmed by both cardiac enzyme and electrocardiographic analyses. The diagnosis of STEMI was based on a suggestive history, with ST elevation $>2 \mathrm{~mm}$ in $\geq 2$ precordial leads, ST elevation $>1 \mathrm{~mm}$ in $\geq 2$ limb leads, or new left branch bundle block on a 12-lead electrocardiogram with a concomitant increase of cardiac markers $\geq$ twice the normal upper limit. We excluded patients who were not available for estimation of glomerular filtration rate (GFR) or who did not complete at least 1 year of follow-up. A final population of 7,679 patients was analyzed in this study.

\section{Assessment of renal function}

Renal function was assessed based on estimated GFR (eGFR). The Chronic Kidney Disease Epidemiology Collaboration (CKD-EPI) equation was used to calculate eGFR in $\mathrm{mL} / \mathrm{min} / 1.73 \mathrm{~m}^{2}$ [14]. The eGFR was categorized as $\geq 90,60-89,30-59$, and $<30 \mathrm{~mL} / \mathrm{min} / 1.73 \mathrm{~m}^{2}$, based on National Kidney Foundation (NKF) Kidney Disease Outcomes Quality Initiative (KDOQI) chronic kidney disease (CKD) stages, but patients with CKD stages 4 and 5 were combined because of relatively small numbers in this category.

\section{Statistical analysis}

Continuous variables with normal distributions are presented as mean \pm standard deviation and compared using Student's t-test. Continuous variables with skewed distributions are presented as median (with $25^{\text {th }}$ and $75^{\text {th }}$ percentiles) and compared using the Mann-Whitney U test. Pearson's $\chi^{2}$ test or Fisher's exact test was used to evaluate differences among categorical variables. Survival analysis after STEMI was estimated using the Kaplan-Meier method to compare survival among groups. Association of renal function and clinical outcomes in men and women was assessed using a Cox proportional hazard model with adjustment for confounders. Analyzed confounders included age, body mass index (BMI), comorbidities (hypertension, diabetes mellitus [DM], ischemic heart disease, hyperlipidemia, smoking status), Killip class $\geq 2$, initial treatment strategies, percutaneous coronary intervention (PCI), angiographic findings, and medical treatments during hospitalization. The interaction between gender and renal function was assessed by entering an interaction term in the Cox proportional hazards model. All statistical tests were 2 -tailed, and $\mathrm{p}<0.05$ was considered significant. Statistical analysis was performed using the Statistical Package for Social Sciences software, version 18.0 (SPSS, IBM, Armonk, NY USA).

\section{Results}

Patients' baseline characteristics are presented in Table 1. A total of 7,679 patients took part in the study, including 5,652 men (age, $59.8 \pm 12.8$ ) and 2,027 women (age, $71.4 \pm 10.0$ ). Women were older and had a higher prevalence of impaired renal function $(20.2 \%$ vs. $38.9 \%, \mathrm{p}<0.001$, defined as eGFR $<60 \mathrm{~mL} / \mathrm{min} / 1.73 \mathrm{~m}^{2}$ ), hypertension, and DM, but had a lower BMI, eGFR, and rate-of-smoking history than men. Serum glucose, total cholesterol, low density lipoprotein cholesterol, and high density lipoprotein cholesterol were higher, while cardiac biomarkers and triglycerides were lower in women. At the time of arrival, women had a lower blood pressure and higher Killip class. Left ventricular ejection fraction did not differ between genders.

With regard to initial treatment strategy, conservative treatment was more commonly performed 


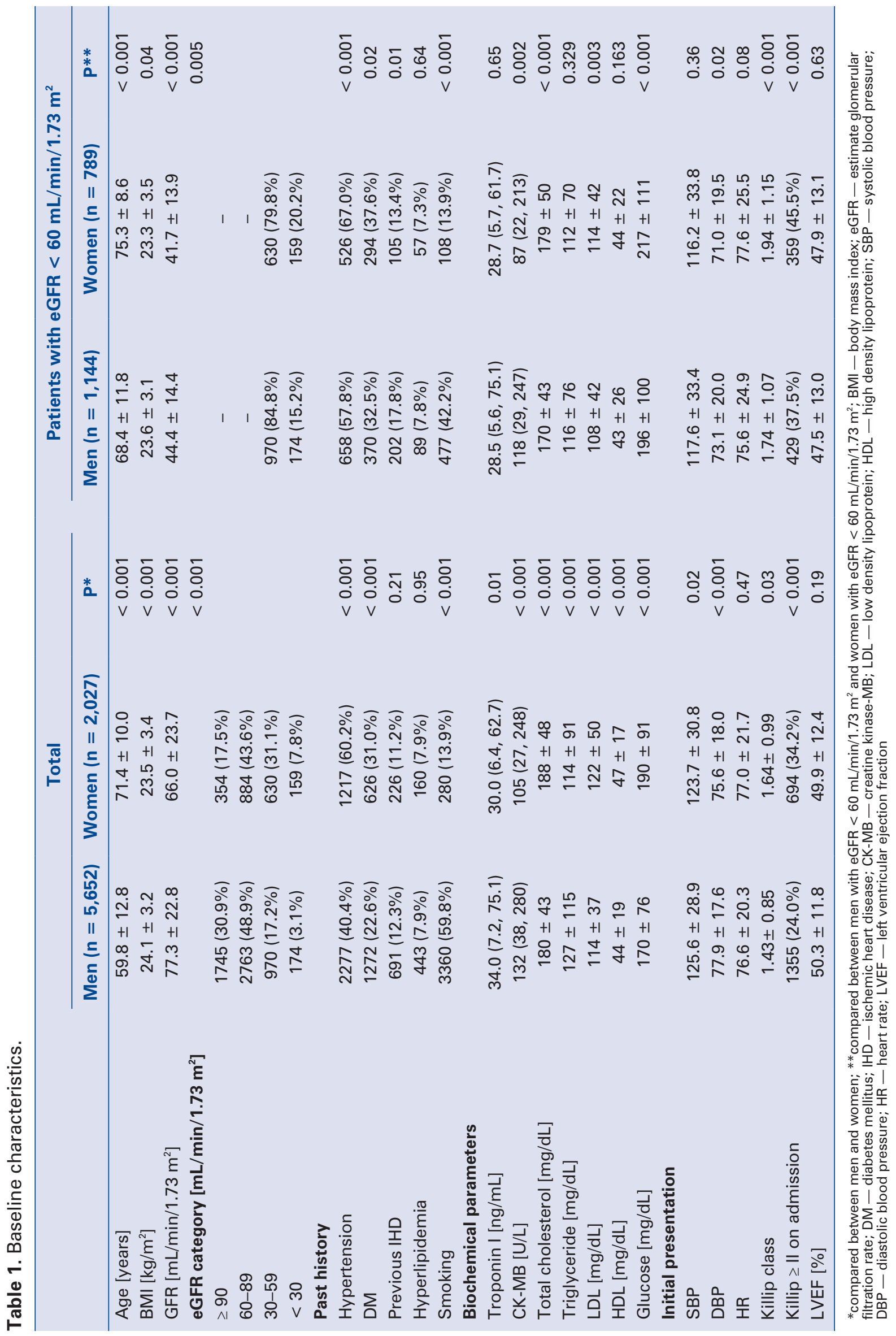


in women, while PCI was undertaken at similar rates in both genders. However, many patients who did not undergo primary PCI underwent PCI before discharge, and overall PCI was less frequently performed in women (Table 2). Women exhibited more multivessel involvement and less optimal post-procedure thrombosis in MI (TIMI) flow on angiographic findings (Table 3). During hospitalization, standard medications known to improve survival after MI, such as anti-platelet agents, beta-blockers, and angiotensin-converting-enzyme inhibitors or angiotensin-receptor-blockers were less frequently used in women, while the use of anti-coagulation drugs and statins did not differ between genders (Table 2).

Subsequently, we analyzed baseline characteristics, hospital treatments and angiographic findings in patients with eGFR $<60 \mathrm{~mL} / \mathrm{min} / 1.73 \mathrm{~m}^{2}$. As showed in all patients, there were similar differences between both genders with regard to baseline characteristics (Table 1), and hospital treatments and angiographic findings did not differ between genders (Tables 2 and 3 ).

Clinical outcomes were assessed by analysis of 1-month and 1-year mortalities. Women showed significantly higher mortality than men after STEMI (1-month mortality, $5.6 \%$ in men vs. $12.6 \%$ in women, $\mathrm{p}<0.001$; 1-year mortality, $6.8 \%$ in men vs. $14.4 \%$ in women, $\mathrm{p}<0.001)$. Kaplan-Meier survival curves with log-rank test showed proportionally increased mortality according to the eGFR category in both genders (Fig. 1). To adjust for possible confounding factors, Cox proportional regression analysis was performed. Decreased renal function was proportionally associated with increased unadjusted 1-month and 1-year mortalities in both genders. After adjusting for potential confounders, eGFR level less than $60 \mathrm{~mL} / \mathrm{min} / 1.73 \mathrm{~m}^{2}$ remained a strong independent predictor for 1 -month and 1-year mortalities in both genders (Table 4). At each eGFR level, hazard ratios for women did not differ significantly from those for men. We performed an interaction test to investigate whether gender influenced mortality at each eGFR level after STEMI. The interaction test showed no significant interaction between gender and eGFR in 1 -month mortality and 1-year mortality $(\mathrm{p}=0.928$, $\mathrm{p}=0.903$, respectively).

\section{Discussion}

In the present study, female STEMI patients were older and had more comorbidity, including impaired renal function, than males, which is consistent with the results of previous studies [4-10].

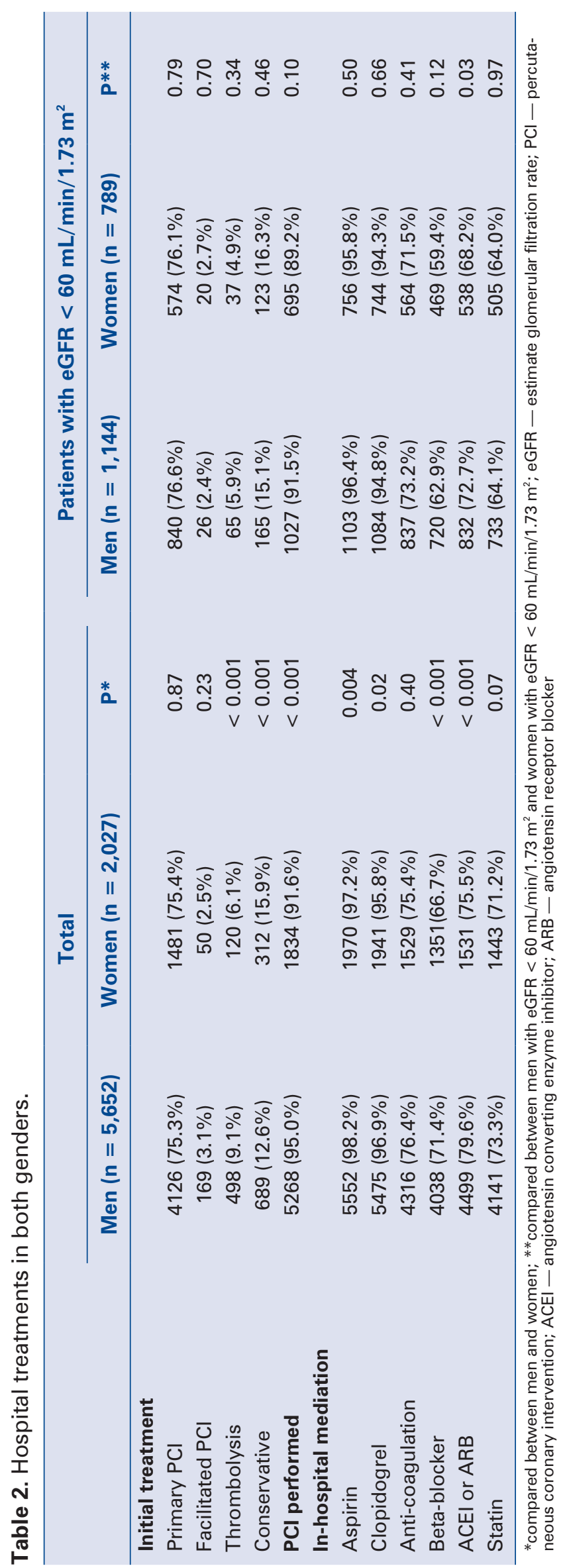


Table 3. Angiographic findings in both genders.

\begin{tabular}{|c|c|c|c|c|c|c|}
\hline & \multicolumn{2}{|c|}{ Total } & \multirow[b]{2}{*}{$\mathbf{P}^{*}$} & \multicolumn{3}{|c|}{ Patients with eGFR $60 \mathrm{~mL} / \mathrm{min} / 1.73 \mathrm{~m}^{2}$} \\
\hline & $\begin{array}{c}\text { Men } \\
\text { (n }=5,652)\end{array}$ & $\begin{array}{c}\text { Women } \\
(n=2,027)\end{array}$ & & $\begin{array}{c}\text { Men } \\
\text { (n }=1,144)\end{array}$ & $\begin{array}{l}\text { Women } \\
(n=789)\end{array}$ & $\mathbf{P} * *$ \\
\hline \multicolumn{2}{|c|}{ Number of diseased vessels } & & $<0.001$ & & & 0.33 \\
\hline Single & $2492(47.6 \%)$ & $732(40.2 \%)$ & & $363(35.2 \%)$ & $223(32.1 \%)$ & \\
\hline Double & $1543(29.5 \%)$ & $581(31.9 \%)$ & & $336(32.6 \%)$ & $229(33.0 \%)$ & \\
\hline Triple & $1075(20.5 \%)$ & $463(25.4 \%)$ & & $288(28.0 \%)$ & $219(31.6 \%)$ & \\
\hline Left main disease & $125(2.4 \%)$ & $45(2.5 \%)$ & & $43(4.2 \%)$ & $23(3.3 \%)$ & \\
\hline Lesion type & & & 0.36 & & & 0.18 \\
\hline A & $214(4.4 \%)$ & $69(4.0 \%)$ & & $41(4.3 \%)$ & $26(4.0 \%)$ & \\
\hline B1 & $869(17.8 \%)$ & $297(17.2 \%)$ & & $144(15.2 \%)$ & $125(19.2 \%)$ & \\
\hline B2 & $1304(26.8 \%)$ & $436(25.3 \%)$ & & $224(23.6 \%)$ & $155(23.8 \%)$ & \\
\hline $\mathrm{C}$ & $2486(51.0 \%)$ & $921(53.5 \%)$ & & $539(56.9 \%)$ & $345(53.0 \%)$ & \\
\hline \multicolumn{2}{|c|}{ Pre procedural TIMI flow grade } & & 0.13 & & & 0.39 \\
\hline 0 & $2633(52.4 \%)$ & $983(55.6 \%)$ & & $540(54.7 \%)$ & $380(56.7 \%)$ & \\
\hline I & $545(10.9 \%)$ & $177(10.0 \%)$ & & $110(11.1 \%)$ & $66(9.9 \%)$ & \\
\hline II & $744(14.8 \%)$ & $252(14.3 \%)$ & & $164(16.6 \%)$ & $95(14.2 \%)$ & \\
\hline III & $1100(21.9 \%)$ & $355(20.1 \%)$ & & $174(17.6 \%)$ & $129(19.3 \%)$ & \\
\hline \multicolumn{2}{|c|}{ Post procedural TIMI flow grade } & & $<0.001$ & & & 0.35 \\
\hline 0 & $98(2.0 \%)$ & $48(2.8 \%)$ & & $31(3.3 \%)$ & $28(4.3 \%)$ & \\
\hline I & $58(1.2 \%)$ & $24(1.4 \%)$ & & $18(1.9 \%)$ & $12(1.8 \%)$ & \\
\hline II & $212(4.3 \%)$ & $134(7.8 \%)$ & & $61(6.4 \%)$ & $54(8.2 \%)$ & \\
\hline III & $4523(92.5 \%)$ & $1514(88.0 \%)$ & & $843(88.5 \%)$ & $562(58.7 \%)$ & \\
\hline
\end{tabular}

*compared between men and women; ${ }^{* *}$ compared between men with eGFR $<60 \mathrm{~mL} / \mathrm{min} / 1.73 \mathrm{~m}^{2}$ and women with eGFR $<60 \mathrm{~mL} / \mathrm{min} / 1.73 \mathrm{~m}{ }^{2}$; eGFR - estimate glomerular filtration rate; TIMI — thrombosis in myocardial infarction
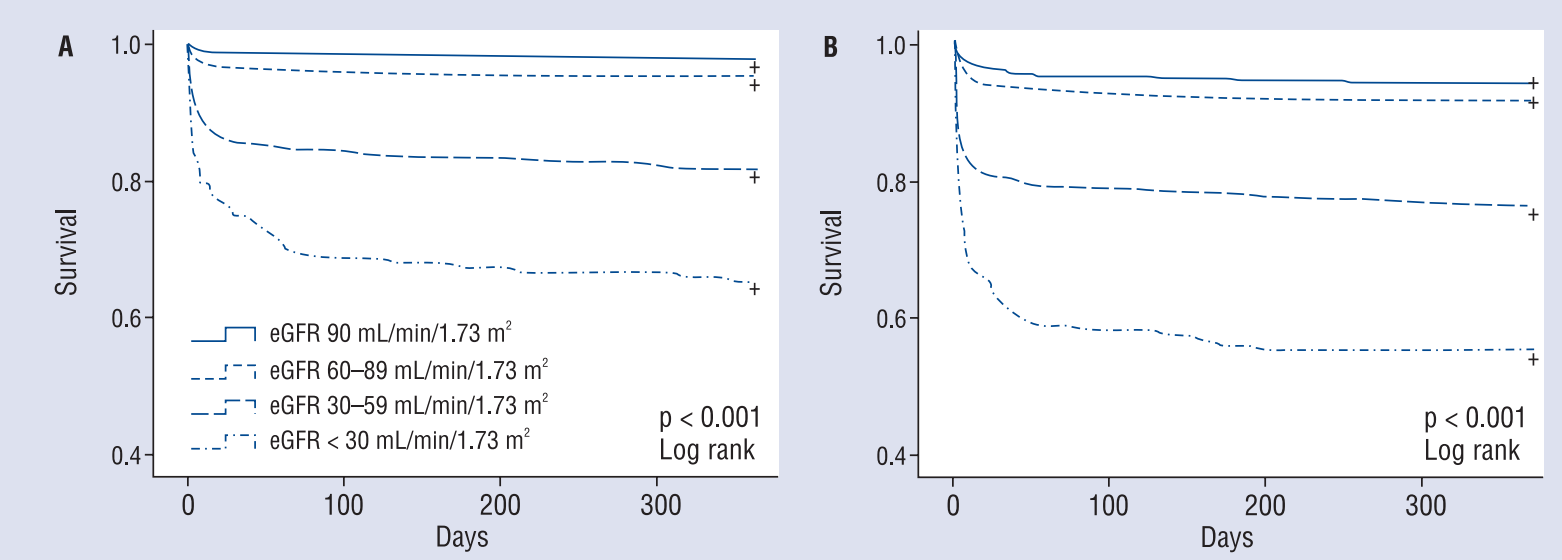

Figure 1. A. One-year mortality Kaplan-Meier curves according to estimated glomerular filtration rate (eGFR) category in men; B. One-year mortality Kaplan-Meier curves according to eGFR category in women.

With regard to hospital management of STEMI, a higher proportion of patients received conservative treatment as an initial treatment strategy, and PCI was less frequently performed during the in-hospital period in women. Indeed, standard medications known to improve survival after MI were less frequently used in women. Although women showed higher mortality in the present study, this might be attributed to differences in baseline characteristics and hospital management intensity. Previous studies reported conflicting results about gender effects on mortality after adjusting 


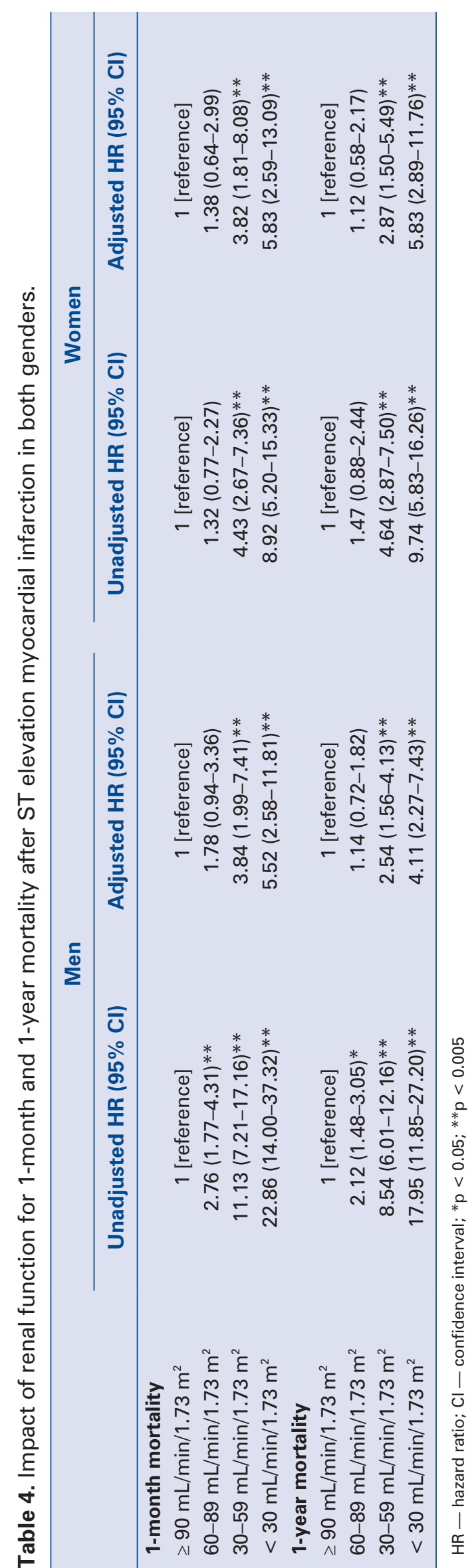

for multiple confounding factors. Some showed no gender effect on mortality after adjustment $[4,5,7,9]$, and others concluded that gender was an independent risk factor for mortality after STEMI $[6,8,10]$. Recently, Kang et al. [9] reported that the gender effect was accounted for mostly by older age in women, complex comorbidities, and severe hemodynamic conditions at presentation; their study analyzed merged data from the KAMIR and the Korean Myocardial Infarction (KorMI) registry.

Previous studies regarding gender effects on the impact of impaired renal function in prognosis after STEMI produced highly discrepant and inconclusive results $[3,11,12]$. In the present study we investigated whether gender influences the impact of impaired renal function in STEMI patients, and we found that the impact of impaired renal function on prognosis after STEMI did not significantly differ between genders. Sederholm Lawesson et al. [12] reported that the association between impaired renal function and outcome after STEMI is stronger in women than in men. In that study no prognostic impact of renal function on clinical outcomes was observed in men; in contrast, in women each $10 \mathrm{~mL} / \mathrm{min} / 1.73 \mathrm{~m}^{2}$ increment of eGFR was associated with $63 \%$ lower risk of death. The interaction test between gender and impaired renal function showed significant differences with respect to mortality. However, we found the opposite in our analysis. The 1-year mortality after STEMI proportionally increased as eGFR decreased, and this impact persisted even after adjusting for potential confounders in both genders. The hazard ratios in each eGFR category were similar in both genders, and the interaction test showed insignificant results. Sederholm Lawesson et al. [12] included an insufficient number of patients (total, 247) to demonstrate a gender effect between impaired renal function and mortality. Chen et al. [3] also reported that gender might influence the prognostic effect of impaired renal function in coronary artery disease patients. In that study they analyzed 1,609 patients enrolled from 1996 to 1997. However, given the recent remarkable advances in treatment of STEMI patients and in quality control, the significance of that study is limited. Recently, Damman et al. [11] have raised questions about previous studies that investigated gender effects on impaired renal function and clinical outcomes. They showed a stronger association between impaired renal function and outcomes in both genders. In contrast to previous studies, they found that hazard ratios at each eGFR category were higher in men than in women, and an interaction test between gender and impaired renal function 
showed significance in overall mortality. Although, Damman et al. [11] analyzed a relatively large number of patients compared to previous studies, the data were based on a single-center-registry, and a significantly higher hazard ratio in men was needed for verification.

Our study has several distinctive advantages compared to previous studies. Firstly, the study population was derived from a multi-center online registry and large-scale cohort populations were analyzed. This is likely to improve statistical reliability. Secondly, the CKD-EPI equation was used to estimate eGFR. This equation was recently described as an improved method of GFR estimation that is more accurate and provides stronger prognostic value than older methods $[14,15]$.

The present study has several limitations. Although we adjusted for multiple confounding factors, it is possible that some unmeasured confounders remain. Moreover, the assessment of kidney function was based on a single serum creatinine value obtained at the time of presentation to the hospital. This value could have been affected by hemodynamic or metabolic status.

\section{Conclusions}

In conclusion, we demonstrated that impaired renal function was an independent prognostic factor after STEMI in both genders, and confirmed that the impact of impaired renal function on prognosis after STEMI did not significantly differ between genders. Our study does not support the notion that gender influences the association between impaired renal function and clinical outcome after STEMI. Our findings broaden current understanding of the role of gender in patients with STEMI.

\section{Acknowledgements}

This research was supported by Basic Science Research Program through the National Research Foundation of Korea (NRF) funded by the Ministry of Education, Science and Technology (2011-0009743), and by the National Research Foundation of Korea (NRF) grant (MRC for Gene Regulation, 2011-0030132) funded by the Korea government (MSIP).

\section{Appendix 1}

Korea Acute Myocardial Infarction Registry (KAMIR) Investigators: Myung Ho Jeong MD, Young Keun Ahn MD, Sung Chull Chae MD, Jong Hyun Kim MD, Seung Ho Hur MD, Young Jo Kim MD, In Whan Seong MD, Dong Hoon Choi MD,
Jei Keon Chae MD, Taek Jong Hong MD, Jae Young Rhew MD, Doo Il Kim MD, In Ho Chae MD, Jung Han Yoon MD, Bon Kwon Koo MD, Byung Ok Kim MD, Myoung Yong Lee MD, Kee Sik Kim MD, Jin Yong Hwang MD, Myeong Chan Cho MD, Seok Kyu Oh MD, Nae Hee Lee MD, Kyoung Tae Jeong MD, Seung Jea Tahk MD, Jang Ho Bae MD, Seung Woon Rha MD, Keum Soo Park MD, Chong Jin Kim MD, Kyoo Rok Han MD, Tae Hoon Ahn MD, Moo Hyun Kim MD, Ki Bae Seung MD, Wook Sung Chung $\mathrm{MD}$, Ju Young Yang MD, Chong Yun Rhim MD, Hyeon Cheol Gwon MD, Seong Wook Park MD, Young Youp Koh MD, Seung Jae Joo MD, Soo Joong Kim MD, Dong Kyu Jin MD, Jin Man Cho MD, Byung Ok Kim MD, Sang-Wook Kim MD, Jeong Kyung Kim MD, Tae Ik Kim MD, Deug Young Nah MD, Si Hoon Park MD, Sang Hyun Lee MD, Seung Uk Lee MD, Hang-Jae Chung MD, Jang Hyun Cho $\mathrm{MD}$, Seung Won Jin, MD, Yang Soo Jang MD, Jeong Gwan Cho, MD, and Seung Jung Park MD.

\section{Conflict of interest: none declared}

\section{References}

1. Anavekar NS, McMurray JJ, Velazquez EJ et al. Relation between renal dysfunction and cardiovascular outcomes after myocardial infarction. N Engl J Med, 2004; 351: 1285-1295.

2. Bae EH, Lim SY, Cho KH et al. GFR and cardiovascular outcomes after acute myocardial infarction: Results from the korea acute myocardial infarction registry. Am J Kidney Dis, 2012; 59: 795-802.

3. Chen R, Kumar S, Timmis A, Feder G, Yaqoob MM, Hemingway H. Comparison of the relation between renal impairment, angiographic coronary artery disease, and long-term mortality in women versus men. Am J Cardiol, 2006; disease, and long- 630 .

4. Cheng CI, Yeh KH, Chang HW et al. Comparison of baseline characteristics, clinical features, angiographic results, and early outcomes in men vs women with acute myocardial infarction undergoing primary coronary intervention. Chest, 2004; 126: 47-53.

5. Fiebach NH, Viscoli CM, Horwitz RI: Differences between women and men in survival after myocardial infarction. Biology or methodology? JAMA, 1990; 263: 1092-1096.

6. Greenland P, Reicher-Reiss H, Goldbourt U, Behar S. In-hospital and 1-year mortality in 1,524 women after myocardial infarction. Comparison with 4,315 men. Circulation, 1991; 83: 484-491.

7. Hirakawa Y, Masuda Y, Uemura K, Kuzuya M, Kimata T, Iguchi A. Differences in in-hospital mortality between men and women with acute myocardial infarction undergoing percutaneous coronary intervention in japan: Tokai acute myocardial infarction study (tamis). Am Heart J, 2006; 151: 1271-1275.

8. Jneid H, Fonarow GC, Cannon CP et al. Sex differences in medical care and early death after acute myocardial infarction. Circulation, 2008; 118: 2803-2810.

9. Kang SH, Suh JW, Yoon CH et al. Sex differences in management and mortality of patients with st-elevation myocardial infarction (from the korean acute myocardial infarction national registry). Am J Cardiol, 2012; 109: acute myoca

10. Tofler GH, Stone PH, Muller JE et al. Effects of gender and race on prognosis after myocardial infarction: Adverse prognosis for women, particularly black women. J Am Coll Cardiol, 1987; 9: 473-482.

11. Damman P, Kikkert WJ, Woudstra P et al. Gender difference in the prognostic value of estimated glomerular filtration rate at admission in ST-segment elevation myocardial infarction: A prospective cohort study. BMJ Open, 2012; 2: e000322

12. Sederholm Lawesson S, Todt T, Alfredsson J, Janzon M, Stenestrand U, Swahn E. Gender difference in prevalence and prognostic impact of renal insufficiency in patients with st-elevation myocardial infarction treated with primary percutaneous coronary intervention. Heart, 2011; 97: 308-314.

13. Kim CS, Choi JS, Park JW et al. Concomitant renal insufficiency and diabetes mellitus as prognostic factors for acute myocardial infarction. Cardiovasc Diabetol, 2011; 10: 95.

14. Levey AS, Stevens LA, Schmid CH et al. A new equation to estimate glomerular filtration rate. Ann Intern Med, 2009; 150: 604-612.

15. Matsushita K, Mahmoodi BK, Woodward M et al. Comparison of risk prediction using the ckd-epi equation and the mdrd study equation for estimated glomerular filtration rate. JAMA, 2012; 307: 1941-1951. 